Published in final edited form as:

Expert Rev Anticancer Ther. 2011 April ; 11(4): 533-540. doi:10.1586/era.10.158.

\title{
Notch signaling in lung cancer
}

\author{
Paola Galluzzo and \\ Loyola University Chicago Medical Center, Oncology Institute, 2160 South First Avenue, \\ Maywood, Illinois, USA, Tel: 708-327-3136, Fax: 708-327-3238, pgalluzzo@lumc.edu
}

\section{Maurizio Bocchetta ${ }^{\dagger}$}

Loyola University Chicago Medical Center, Department of Pathology and Oncology Institute, 2160

South First Avenue, Maywood, Illinois, USA, Tel: 708-327-3362, Fax: 708-327-3238, mbocche@lumc.edu

\section{Summary}

Lung cancer is the leading cause of cancer-related deaths in the Western world. Lungs can be affected by a number of histologically diverse malignancies. Nonetheless, the vast majority of lung cancers are classified as non-small cell lung cancer (NSCLC). Despite extensive research on different therapeutic regimens, the overall 5-year survival of patients diagnosed with NSCLC (all stages) is a dismal $15 \%$. Although strongly correlated with tobacco smoke, there is an increasing NSCLC morbidity in individuals who have never smoked. The pattern of genetic lesions found in NSCLC derived from smokers and never-smokers appears to be different. This fact led to the hypothesis that different, still unidentified carcinogens are responsible for lung cancer onset in never-smokers. All the above considerations compel the scientific community to find novel therapeutic targets to fight such a deadly disease.

In recent years critical pathways governing embryonic development have been increasingly linked to cancer. Here we will focus on the role of Notch signaling in lung cancer. Notch receptors' activity can be blocked following different strategies, thus representing a promising alternative/ complement to the arsenal of therapeutic strategies currently used to treat lung cancer.

\section{Keywords}

lung cancer; cancer progenitor cells; Notch signaling; hypoxia; cancer stem cells; Notch signaling inhibition

\section{Lung cancers and their progenitor cells}

Lung cancers are a group of different histopathological malignancies. The most frequently diagnosed lung carcinomas are histologically classified as small cell lung carcinoma (SCLC) and non-small cell lung carcinoma (NSCLC). SCLC is an extremely aggressive malignancy that accounts for $13-15 \%$ of all lung neoplasms in the USA [1,2]. SCLC is of neuroendocrine origin and is the lung malignancy most associated with cigarette smoking [2]. Given its highly proliferative/dissemination rate, the vast majority of SCLC patients present with extensive stage disease. SCLC patients with limited stage disease who receive current therapeutic regimens can achieve a five-year survival of about $14 \%$ [3].

\footnotetext{
${ }^{\dagger}$ Author for correspondence, Loyola University Chicago Medical Center, Department of Pathology and Oncology Institute, 2160 South First Avenue, Maywood, Illinois, USA, Tel: 708-327-3362, Fax: 708-327-3238, mbocche@lumc.edu.
} 
NSCLC accounts for virtually the rest of lung cancer cases. It is broadly subdivided into three major histological classifications: squamous cell carcinoma (SCC, which is, similarly to SCLC, the histology subtype most associated with smoking), large cells carcinoma (LCC) and adenocarcinoma of the lung (ACL), which accounts for the majority of cases of NSCLC and it is the most frequent lung malignancy in individuals who have never smoked [4].

SCLC and SCC are generally considered bronchogenic, while LCC and ACL are generally initiated at the periphery of the lungs [5,6] (see Figure 1 for a schematic representation of the airways epithelia). Given their differential location of origin, it is likely that the different histological types of lung cancer initiate from genetic damage of distinct stem/precursor cells.

It is generally hypothesized that stem cells have features that makes them ideal cancerinitiating cells. Stem cells are long lived because of their capacity of self-renewal. A longlived cell has more chances to accrue the number of disparate genetic damage that ultimately lead to malignant transformation. Stem or progenitor cells also have the feature of extensive proliferative potential through transit-amplifying daughter cells [7]. It should be noted that a progenitor cell, in order to initiate the process of repopulation/differentiation, must reactivate molecular pathways that play an essential role during embryonic development, such as Notch signaling. A genetically damaged progenitor cell that becomes unable to "turn off" these pathways will eventually develop into a tumor. In the past two decades there has been a growing body of evidence underscoring the importance of developmental pathways (such as Wnt, Hedgehog, Notch) in carcinogenesis. This emphasizes the intimate connection between tumorigenesis and developmental processes, a concept first proposed by Rudolf Virchow in 1858 [8]. For example, malignant tissues appear undifferentiated, the increased motility of cancer cells is reminiscent of the processes of gastrulation and organogenesis, some malignancies show embryonic tissue organization, etc.

Contrary to the intestine, the lung is not an organ where continuous renewal of the epithelium takes place. Nonetheless, lung epithelia can repair after injury. Some studies have identified "side populations" as per Hoechst stain efflux in the lung, similar to what observed in the hematopoietic system [9]. Lung regeneration studies have identified distinct multipotent progenitor cells in different districts of the lung that can initiate this process of repair. In the bronchi, progenitor cells capable of regenerating the airway epithelium have been identified in a number of studies involving mechanical or chemical damage. The consensus appears to point out to a subset of non-ciliated, basal cells that do not merely provide support to the pseudostratified epithelium of trachea and bronchi. Focus has been concentrated to naphthalene-resistant Clara cell-like populations, which appear absolutely necessary for bronchial regeneration [10]. A study conducted using a variety of injuring strategies identified additional, pluripotent basal cell subpopulations expressing cytokeratin 14 that can regenerate damaged bronchial epithelia [10]. It is conceivable that these basal cells, alongside with calcitonin gene-related peptide (CGRP)-expressing pulmonary neuroendocrine cells (PNECs) may represent cancer-initiating cells for those malignancies that originate in the bronchi.

There has been intense debate concerning the progenitor cells for distally-originated lung malignancies (such as ACL). ACL displays markers that are both characteristic of T2 pneumocytes, such as prosurfactant apoprotein-C (SP-C) and of Clara cells (Clara cell secretory protein, or CCSP). Naphthalene-resistant, Clara-like cells capable of repopulating damaged terminal bronchioles have been identified at the junction of terminal bronchioles and alveoli (bronchio-alveolar duct junction or BADJ) [11]. Further investigations identified that within the BADJ there is a limited number of cells positive for both SP-C and CCSP (therefore displaying markers of both Clara cells and T2 pneumocytes). These cells are 
capable to start proliferating after alveolar or bronchiolar injury and are responsible for alveolar repopulation after damage in a hierarchical order (T2, following transdifferentiation to T1 pneomocytes). These cells have been defined as bronchioalveolar stem cells (BASC) [12]. Oncogenic RAS activation together with chemical insult of mice lungs leads to a series of events: expansion of the BASC population, atypical adenomatous hyperplasia of the lung, adenomas and ultimately ACL [12]. These data strongly implicate BASCs as the cells of origin of ACL.

\section{Current therapeutic strategies for lung cancer and their limitations}

A detailed list of therapeutic strategies for lung cancer according to stage and tumor presentation can be found elsewhere [5,6]. As an oversimplification, NSCLC is treated with surgery, different modalities of chemotherapy and radiation therapy. However, after four decades of research, the five-year survival rate for the types of lung cancer discussed here has modestly improved since the introduction of cisplatin. This is because, even in responsive patients, the malignancy will eventually recur and become therapy insensitive (of interest, in several malignancies resistance to platinum-based therapies has been attributed to enhanced Notch-1 signaling, [13]). The reasons for recurrence have been attributed to different factors. For very early stage NSCLC, de novo malignant growth after surgical resection can be attributable to "field cancerization" [14]. It is generally known that the airway epithelium of individuals exposed to carcinogens has acquired a number of genetic damages, which renders it susceptible for the development of a subsequent carcinoma. For instance, even in smokers without lung cancer, several preneoplastic areas have been detected, which persist for decades even after smoking cessation [15]. It should be stressed that modern molecular research has rendered the notion that recurrences may be the result of micrometastases or intraepithelial migration of resected tumor cells rather unlikely.

Chemotherapy resistance appears the result of the existence of a subpopulation of cancer cells with distinct features compared to the bulk of the tumor mass. They were first identified as clonogenic cells that were able to induce tumors similar to those of the original patient when injected intracranially in nude mice. These "lung Cancer Stem Cells" (CSC) have properties similar to those first identified in hematopoietic malignancies. CSC are cells with distinct characteristics: they are able of self renewal. After one round of cell division, a CSC will produce two cells: one maintaining stem status, and a second that can differentiate into a rapidly dividing cell, which will ultimately generate the bulk of the tumor mass. Lung cancer CSC have not yet been clearly identified in terms of expression of specific markers. Their existence can be inferred from their properties (other than their clonogenicity) common to better-characterized CSC.

CSC overexpress ATP-binding transporters [16] hence they are able to rapidly remove drugs from their cellular environment. For this characteristic, they can be identified and isolated as side-populations with low Hoechst 33342 dye staining. A recent study identified such side populations in all six NSCLC cell lines and in 16 surgically resected NSCLC analyzed. These side populations were highly oncogenic, were able to reconstitute (in culture) cellular populations similar of those before cell sorting, and were resistant to seven commonly used anticancer drugs [17].

Besides their ability of rapid efflux of drugs from the cell, CSC are a difficult target for anticancer therapy because they are quiescent or divide infrequently, a feature that can be measured by bromodeoxyuridine labeling techniques [12]. More importantly, CSC reside in hypoxic tumor microenvironment [18]. Solid tumors are intrinsically hypoxic, and hypoxia is a critical factor of radio/chemotherapeutic resistance [19]. Tumor growth outpaces the capability of neovascularization. The high levels of vascular endothelial growth factor 
(VEGF) released by cancer cells and stroma lead to architecturally inefficient new blood vessels, with vascular leakage that further reduces the delivery of oxygen, nutrients and chemotherapeutic agents to cancer cells [20]. Hypoxia leads to stabilization of hypoxia inducible factor $2 a$ (HIF-2a), which increases the cellular capability of DNA damage repair $[21,22]$. HIF-1 $a$, through its interplay with Notch-1 under hypoxia, activates the major antiapoptotic pathways of the cell in hypoxic ACL microenvironment [23]. Notch signaling pathway seems to play a pivotal role in maintaining the undifferentiated state and the number of stem and progenitor cells [24,25]. For example, in breast cancer, Notch-4 and Notch-1 inhibition reduces CSC tumorigenicity [26].

$\mathrm{CSC}$ are also responsible for rapid cancer repopulation after radio/chemotherapy. This property could open a window for the eradication of CSC, therefore achieving complete cure of residual disease [20].

\section{Notch signaling in brief}

Notch are an evolutionarily conserved family of transmembrane receptors (Notch-1 through -4 in mammalians) regulating critical cell fate decisions during development and post-natal life [27]. Notch receptors are heterodimeric proteins, consisting of a large, modular Nterminal extracellular portion (Notch ${ }^{\mathrm{EC}}$ ) non-covalently bound to a trasmembrane domain $\left(\right.$ Notch $\left.^{\mathrm{TM}}\right)$, followed by an intracellular, C-terminal portion. After their synthesis, Notch precursors are cleaved in the trans-Golgi by a furin-like convertase (at cleavage site 1, or S1), giving rise to mature proteins, which are exposed on the plasma membrane, where they can interact with their ligands [27]. In humans, these consist of two Serrate-like proteins (Jagged-1 and -2), and a Delta-like protein family (see Figure 2 for details). Preferential binding of each individual ligand to Notch receptors has not yet been clarified, but it could be modulated by the protein glycosylases FRINGE and RUMI, which modifies Notch receptors through their maturation $[27,28]$. Notch ligands are single-pass transmembrane proteins that interact with Notch when presented to Notch by a neighboring cell. According to this model, a signaling cell presents ligands to a juxtaposed Notch expressing cell.

Upon ligand binding, Notch ${ }^{\mathrm{EC}}$ (essentially an inhibitory domain) is trans-endocyted and pulled away from Notch ${ }^{\mathrm{TM}}$ [29]. At this stage Notch ${ }^{\mathrm{TM}}$ undergoes two proteolitic cleavages. The first is operated (at S2) by A-disintegrin and metalloprotease (ADAM) 10 or 17 (or both, since ADAM-17 cleaves Notch ${ }^{\mathrm{TM}}$ in vitro, but only ADAM-10 knockout mice display a Notch phenotype). S2 cleavage releases the extracellular stump of Notch ${ }^{\mathrm{TM}}$, triggering a third proteolytic cleavage operated by a $\gamma$-secretase complex that cuts Notch proteins within the transmembrane sequence (S3). Where exactly $\gamma$-secretase cleavage takes place (e.g., plasma membrane or endocytic vesicles) is still a matter of debate. More so the faith of cleaved Notch, which can be rapidly directed to proteosomal degradation when associated with NUMB [reviewed in 27]. According to a general consensus, S3 cleaved Notch are quite unstable proteins (however, Notch- $1^{\mathrm{IC}}$ is stabilized by HIF-1a in hypoxic conditions $[24,30])$. The ultimate event in Notch multiple proteolytic processing leads to the release in the nucleus of the activated form of Notch (intracellular Notch, or Notch ${ }^{\mathrm{IC}}$ ). In the nucleus Notch ${ }^{\mathrm{IC}}$ modulates gene expression primarily by binding to the ubiquitous transcription factor CBF-1. Therefore, the inhibition of $\gamma$-secretase activity in turn leads to suppressed Notch signaling. Notch activation does not necessarily require the interaction of two distinct cells. Some cells express both Notch receptors and their ligands. Moreover, a study suggests that Notch proteins can interact with their ligands within the cytoplasm [31].

The binding of Notch ${ }^{\mathrm{IC}}$ to CBF-1 dissociates a co-repressor complex including SMRT (silencing mediator of retinoid and thyroid hormone receptors), the co-repressor CIR, histone deacetylase I (HDAC-1), SHARP (SMRT/HDAC-associated repressor protein), 
CtIP/CtBP corepressors. Notch/CBF-1 complexes recruit nuclear co-activators such as SKIP, Mastermind-like proteins, histone acetyl transferases p300, CPB associated factor (PCAF) and GCN5 [27]. Therefore, the net result of the Notch ${ }^{\mathrm{IC}}-\mathrm{CBF}-1$ interaction is the conversion of CBF-1 from a transcription repressor to an activator of transcription for a number of helix-loop-helix transcriptional repressors collectively named as Enhancer of Split [E(spl)] in Drosophila, or HES, HEY and HERP genes in humans [27]. Other CBF-1/ Notch targets are the $\mathrm{p} 21^{\mathrm{WAF} 1}$ promoter [27,32], cyclin D1, cyclin A, members of the NF$\kappa \mathrm{B}$ pathway, and the ubiquitin ligase SKP2, which in turn promotes the degradation of the cyclin dependent kinase inhibitor p27 ${ }^{\mathrm{KIP} 1}$ [27], c-myc [33], insulin-like growth factor 1 (IGF-1) and its receptor (IGF-1R) [23].

Activation of $E(s p l)$ causes repression of differentiation factors. Notch activation signals to the cell the maintenance of a pre-committed state, which allows it to properly interpret differentiation or proliferation stimuli, and its self-renewal in the progenitor compartment. During post-natal life, Notch regulation of cell proliferation, differentiation and apoptosis is context dependent. Notch-1 induces growth arrest and promotes differentiation of keratinocytes. Forced overexpression of Notch-1 inhibits proliferation of myeloid progenitor cells, and causes growth arrest in B cells. Conversely, Notch signaling promotes proliferation in hematopoietic progenitor cells, promotes murine hematopoietic stem cell self-renewal and stimulates proliferation of mammary epithelial cells. Notch induction triggers apoptosis in B cells, but has antiapoptotic effects in T cell lines, and in murine erythroleukemia cells [27].

Notch receptors activation display redundant and distinct features. Only Notch-1 knockout mice and mice homozygous for mutant Notch-2 are embryonically lethal at around E11 $[34,35]$. Notch-1 knockouts had "widespread cell death" in all tissues, while in the Notch-2 mutants cell death was mostly pronounced in neural tissues. These data would indicate that both Notch-1 and -2 signaling are essential for cell survival. However, there are reports indicating that Notch-1 and Notch-2 activities can have opposite biologic outputs in some tissues [32,36]. During lung development Notch-1 is expressed in airway epithelium undergoing rapid proliferation and is thought to direct differentiation of the epithelial component of the lung. Notch-2 is expressed in lung mesenchyme, Notch-3 is expressed in both epithelial and mesenchymal compartments, while Notch-4 appears to be endothelialspecific. No Notch receptor is expressed in neuroendocrine cells [37].

\section{Notch signaling in lung cancer}

Notch signaling is increasingly linked to a variety of malignancies [reviewed in [27]. In lung cancer two Notch paralogs have been implicated: Notch-1 and Notch-3. No study has highlighted a specific role for Notch-2, Notch-4, or deregulation of Notch ligands expression.

\section{The Notch-1 connection}

In an early study, overexpression of Notch- ${ }^{\mathrm{IC}}$ caused growth arrest of SCLC cell lines [38], thus suggesting a possible tumor suppressive role for Notch-1 in SCLC. Such concept is in line with what observed in other neuro-endocrine tumors $[39,40]$.

The role for Notch-1 in NSCLC has been initially controversial. However, more recent studies from different groups have converged into the notion that Notch-1 may be an important oncogene in NSCLC. The Yi group showed that forced expression of constitutively active Notch-1 inhibited growth of the ACL line A549 cultured in standard conditions and interfered with tumor growth in vivo [41]. We have obtained analogous results in the same and other NSCLC cell lines cultured in normoxia [30]. However, 
experiments generated from Notch-1 ${ }^{\mathrm{IC}}$ overexpression only are difficult to interpret and may lead to artifactual results. Indeed, dissimilar conclusions were proposed by a Chinese study based on immunohistochemical analysis of NSCLC tumor biopsies compared to normal "bronchi mucosa". These authors observed strong Notch-1 immunoreactivity in NSCLC compared to normal lung, which correlated with Jagged-1 and VEGF expression. The intensity of the immunostaining increased in more advanced tumor stages [42].

In a recent study, downregulation of NUMB was detected in about 30\% of NSCLC; this led to elevated Notch-1 activity. The same study found Notch-1 activating mutations in about $10 \%$ of NSCLC, and these mutations conferred a worse prognosis in patients with wt p53 [43].

We have found that Notch-1 provides critical survival signals to NSCLC cells specifically under hypoxia. Inhibition of Notch-1 signaling in NSCLC cells (obtained either through genetic downregulation or using a $\gamma$-secretase inhibitor, or GSI) caused apoptosis under hypoxia only [30]. GSI toxicity could be reversed by forced expression of Notch-1 ${ }^{\mathrm{IC}}$. Immunohistochemical analysis of frozen ACL specimens compared to matching normal lung showed an inconsistent expression of Notch- $1^{\mathrm{IC}}$ in tumor samples compared to normal lung epithelia. Further studies revealed that Notch- $1^{\mathrm{IC}}$ expression was limited to tumor hypoxic microenvironment only [23]. Notch-1 signaling in hypoxic NSCLC regions appears to promote cancer cell survival by a dual mechanism: inhibition of phosphatase and tensing homolog (PTEN) expression and positive regulation of IGF-1 and its receptor IGF-1R [23]. From these data, Notch-1 inhibition could represent an invaluable strategy to target hypoxic tumor regions, which are resistant to standard chemotherapy and provide a nice to CSC.

A recent study provided additional evidence that Notch-1 may exert pro-oncogenic functions in NSCLC. Baumgart et al. [44] found that in NSCLC ADAM17 promotes EGF-R independently from its role in EGF shedding from its membrane-anchored precursor. The target of ADAM-17 was found to be Notch-1. The authors described a positive regulation of Notch-1 of the EGF-R expression levels. Concurrent activation of Notch-1 and EGF-R (through its ligand) supported NSCLC cell survival and promoted colony formation. In archival human NSCLC specimens (but not in normal lung) significant correlation between ADAM-17, Notch-1 and EGFR overexpression was found. From their findings the authors proposed a molecular circuitry that involves ADAM-17 mediated over-activation of Notch-1, which in turn positively regulates EGF-R expression. ADAM-17 overexpression increases the processing of the EGF precursor, thereby feeding into a positive feedback loop that promotes the malignant phenotype [44].

\section{The role of Notch-3}

An oncogenic role for Notch-3 in about a third of NSCLC cases has been proposed. In an early study a chromosomal translocation $\mathrm{t}(15 ; 19)$ was found in a lung carcinoma [45]. Similar translocations were detected in two separate thoracic malignancies including a thymic carcinoma [46-48]. The gene to be affected by such translocation was proposed to be Notch-3 because the NSCLC cell line HCC2429 (harboring such translocation) expressed high levels of the Notch-3 mRNA. Moreover, 7 out of 44 NSCLC cell lines expressed the Notch-3 mRNA as determined by Northern blot hybridization [45]. A more recent study showed that the expression of a truncated Notch-3 receptor (missing the intracellular portion of the protein, and therefore considered as a Notch-3 dominant negative form) caused reduced growth of NSCLC HCC2429 and H460 cell lines and increased "growth factor dependence" [49]. The same study also analyzed 207 resected lung cancers (including 10 lung carcinoids) by immunohistochemistry, and found Notch-3 expression in 80 specimens. The highest frequency of expression was found in squamous cell carcinomas and 
adenocarcinomas (45\% and 37\%, respectively) and the expression of Notch-3 in these tumors was significantly correlated to epidermal growth factor receptor (EGF-R). Cooperation between Notch-3 and EGF-R in NSCLC cell lines HCC2429 and H460 has been recently proposed [50]. Genetic downregulation of Notch-3 led to induction of the proapoptotic, BH3-only polypeptide BIM. The latter is a well-known mediator of gefitinib toxicity in NSCLC with activating mutations of the EGF-R [51]. GSI MRK-003 treatment in combination with erlotinib drastically reduced xenograft growth of the cell line H460 [52].

Treatment of mice with the MRK-003 reduced tumor formation in xenografts mouse models injected with HCC2429 and $\mathrm{H} 460$ cell lines, while the effect was minimal in animals injected with A549 cells. If Notch-3 was genetically inhibited through RNA interference, the same cells became GSI insensitive, indicating that Notch-3 was the target of the GSI used [52]. However, the latter observation seems somewhat confusing since, in our view, the inhibition of a given oncogene (in this case Notch-3), should produce the same phenotypic outcome independently of the method used to achieve this inhibition (i.e., post-

transcriptional downregulation or chemical blockage). This concept is reinforced by a recent study where the Dang group screened a peptide library spanning the entire extracellular portion of Notch-3, identifying a number of peptides (corresponding to EGF repeats 7-10 and 21-22) which bound to Jagged-1, decreased HEY-1 expression, promoted HCC2429 cell line apoptosis and decreased tumorigenicity in vivo [53].

A role for Notch-3 in NSCLC pathogenesis is also supported by transgenic mice studies. Mice expressing Notch-3 $3^{\mathrm{IC}}$ under the SP-C promoter displayed lack of T1 pneumocyte differentiation, reduced T2 pneumocytes number, with the majority of the cells lining the developing alveolar space being undifferentiated [54]. In our opinion, the amount of data described above support the notion that a fraction of NSCLC requires Notch-3 signaling for proliferation and survival.

\section{Expert commentary}

Notch signaling is emerging as a potentially invaluable molecular pathway as a target for novel NSCLC treatment options, especially for its role played in hypoxic tumor environment. Notch can be inhibited using different strategies. ADAM inhibitors are in preclinical development. Different GSIs are already being tested in 4 phase I clinical trials. Stabilized peptides that interfere with receptor/ligand interactions could well serve as an alternative [53]. Recently, cell permeable, stabilized a-helical peptides that can interfere with the function of the Notch transactivation domain have been described [55]. Another strategy is aimed at targeting individual Notch receptors using antibodies that "lock" the negative regulatory region in such a way that ADAM protease cannot have access at cleavage site 2 of Notch receptors, thus preventing the initiation of different Notch receptors activation [56]. This last strategy, although potentially "expensive", may circumvent gut toxicity caused by pan-Notch inhibitors such as GSI or ADAM inhibitors [57].

\section{Five-year view}

Notch inhibition for the treatment of solid tumors, including NSCLC, will inevitably grow in the near future. Initially developed to treat Alzheimer disease, GSIs appear in a more advanced stage and they already entered the clinic in ongoing phase I clinical trials. In our opinion, it is unlikely that Notch-targeting drugs will be effective anticancer instruments if used as single agents. The next challenge for the field will be to find the most effective "doublet" to maximize the potential of Notch inhibitors. 


\section{Acknowledgments}

Support. NCI - R01 CA134503 (M.B.)

\section{References}

1. Rosti G, Bevilacqua G, Bidoli P, Portalone L, Santo A, Genestreti G. Small cell lung cancer. Ann. Oncol. 2006; 17(Suppl 2ii):5-10. [PubMed: 16143594]

2. Govindan R, Page N, Morgensztern D, et al. Changing epidemiology of small-cell lung cancer in the United States over the last 30 years: analysis of the surveillance, epidemiologic, and end results database. J. Clin. Oncol. 2006; 24(28):4539-4544. [PubMed: 17008692]

3. Jänne PA, Freidlin B, Saxman S, et al. Twenty-five years of clinical research for patients with limited-stage small cell lung carcinoma in North America. Cancer. 2002; 95(7):1528-1538. [PubMed: 12237922]

4. Shigematsu H, Lin L, Takahashi T, et al. Clinical and biological features associated with epidermal growth factor receptor gene mutations in lung cancers. J. Natl. Cancer. Inst. 2005; 97(5):339-346. [PubMed: 15741570] . ** Description of EGF-R activating mutations found specifically in adenocarcinoma of the lung in never smokers versus ever smokers, East Asian ethnicity versus other ethnical groups, and female versus male patients.

5. Schrump, DS.; Giaccone, D.; Kelsey, CR.; Marks, LB. Non-Small-Cell Lung Cancer. In: De Vita, VT., Jr; Lawrence, TS.; Rosemberg, SA., editors. Cancer, Principles \& Practice of Oncology. 8th Edition. Philadelphia, PA: Lippincot Williams \& Wilkins; 2008. p. 896-946.

6. Krug, LM.; Kris, MG.; Rosenzweig, K.; Travis, WD. Small Cell and Other Neuroendocrine Tumors of the Lung. In: De Vita, VT., Jr; Lawrence, TS.; Rosemberg, SA., editors. Cancer, Principles \& Practice of Oncology. 8th Edition. Philadelphia, PA: Lippincot Williams \& Wilkins; 2008. p. 946-971.

7. Weissman IL, Anderson DJ, Gage F. Stem and progenitor cells: origins, phenotypes, lineage commitments, and transdifferentiations. Annu. Rev. Cell. Dev. Biol. 2001; 17:387-403. [PubMed: 11687494]

8. Virchow, RLK. Cellular Pathology. Berlin: 1859.

9. Giangreco A, Shen H, Reynolds SD, Stripp BR. Molecular phenotype of airway side population cells. Am. J. Physiol. Lung. Cell. Mol. Physiol. 2004; 286(4):L624-L630. [PubMed: 12909587]

10. Hong KU, Reynolds SD, Watkins S, Fuchs E, Stripp BR. Basal cells are a multipotent progenitor capable of renewing the bronchial epithelium. Am. J. Pathol. 2004; 164(2):577-588. [PubMed: 14742263] . Conclusive identification of the main progenitor cells for maintenance of the bronchial airway epithelium.

11. Giangreco A, Reynolds SD, Stripp BR. Terminal bronchioles harbor a unique airway stem cell population that localizes to the bronchoalveolar duct junction. Am. J. Pathol. 2002; 161(1):173182. [PubMed: 12107102]

12. Kim CF, Jackson EL, Woolfenden AE, et al. Identification of bronchioalveolar stem cells in normal lung and lung cancer. Cell. 2005; 121(6):823-835. [PubMed: 15960971] . ** Identification of adenocarcinoma of the lung initiating cells, also responsible for alveolar repair after injury.

13. Meng RD, Shelton CC, Li YM, et al. gamma-Secretase inhibitors abrogate oxaliplatin-induced activation of the Notch-1 signaling pathway in colon cancer cells resulting in enhanced chemosensitivity. Cancer Res. 2009; 69(2):573-582. [PubMed: 19147571]

14. Slaughter DP, Southwick HW, Smejkal W. Field cancerization in oral stratified squamous epithelium; clinical implications of multicentric origin. Cancer. 1953; 6(5):963-968. [PubMed: 13094644]

15. Wistuba II, Lam S, Behrens C, et al. Molecular damage in the bronchial epithelium of current and former smokers. J. Natl. Cancer. Inst. 1997; 89(18):1366-1373. [PubMed: 9308707]

16. Bunting KD. ABC transporters as phenotypic markers and functional regulators of stem cells. Stem Cells. 2002; 20(1):11-20. [PubMed: 11796918]

17. Ho MM, Ng AV, Lam S, Hung JY. Side population in human lung cancer cell lines and tumors is enriched with stem-like cancer cells. Cancer Res. 2007; 67(10):4827-4833. [PubMed: 17510412] 
18. Keith B, Simon MC. Hypoxia-inducible factors, stem cells, and cancer. Cell. 2007; 129(3):465472. [PubMed: 17482542]

19. Brown JM. Tumor hypoxia in cancer therapy. Methods Enzymol. 2007; 435:297-321. [PubMed: 17998060]

20. Milas L, Hittelman WN. Cancer stem cells and tumor response to therapy: current problems and future prospects. Semin. Radiat. Oncol. 2009; 19(2):96-105. [PubMed: 19249647]

21. Bao S, Wu Q, McLendon RE, et al. Glioma stem cells promote radioresistance by preferential activation of the DNA damage response. Nature. 2006; 444(7120):756-760. [PubMed: 17051156]

22. Li Z, Bao S, Wu Q, et al. Hypoxia-inducible factors regulate tumorigenic capacity of glioma stem cells. Cancer Cell. 2009; 15(6):501-513. [PubMed: 19477429] . * Demonstration that HIF-2a is necessary for glioma cancer stem cells self-renewal, proliferation, and survival in vitro and in vivo.

23. Eliasz S, Liang S, Chen Y, et al. Notch-1 stimulates survival of lung adenocarcinoma cells during hypoxia by activating the IGF-1R pathway. Oncogene. 2010; 29(17):2488-2498. [PubMed: 20154720]

24. Gustafsson MV, Zheng X, Pereira T, et al. Hypoxia requires notch signaling to maintain the undifferentiated cell state. Dev Cell. 2005; 9(5):617-628. [PubMed: 16256737] . ** Demonstration of the interplay between HIF-1 $a$ and Notch-1 in preventing differentiation. First evidence that oxygen levels quantitatively and qualitatively affect Notch-1 signaling.

25. Androutsellis-Theotokis A, Leker RR, Soldner F, et al. Notch signaling regulates stem cell numbers in vitro and in vivo. Nature. 2006; 442(7104):823-826. [PubMed: 16799564] . ** Demonstration of neural stem cell expansion in vitro and in vivo after Notch stimulation.

26. Harrison H, Farnie G, Howell SJ, et al. Regulation of breast cancer stem cell activity by signaling through the Notch4 receptor. Cancer Res. 2010; 70(2):709-718. [PubMed: 20068161]

27. Miele L. Notch signaling. Clin. Cancer Res. 2006; 12(4):1074-1079. [PubMed: 16489059]

28. Acar M, Jafar-Nejad H, Takeuchi H, et al. Rumi is a CAP10 domain glycosyltransferase that modifies Notch and is required for Notch signaling. Cell. 2008; 132(2):247-258. [PubMed: 18243100]

29. Gordon WR, Vardar-Ulu D, Histen G, Sanchez-Irizarry C, Aster JC, Blacklow SC. Structural basis for autoinhibition of Notch. Nat. Struct. Mol. Biol. 2007; 14(4):295-300. [PubMed: 17401372]

30. Chen Y, De Marco MA, Graziani I, et al. Oxygen concentration determines the biological effects of NOTCH-1 signaling in adenocarcinoma of the lung. Cancer Res. 2007; 67(17):7954-7959. [PubMed: 17804701]

31. Sakamoto K, Ohara O, Takagi M, Takeda S, Katsube K. Intracellular cell-autonomous association of Notch and its ligands: a novel mechanism of Notch signal modification. Dev. Biol. 2002; 241(2):313-326. [PubMed: 11784114]

32. Graziani I, Eliasz S, De Marco MA, et al. Opposite effects of Notch-1 and Notch-2 on mesothelioma cell survival under hypoxia are exerted through the Akt pathway. Cancer Res. 2008; 68(23):9678-9685. [PubMed: 19047145]

33. Weng AP, Millholland JM, Yashiro-Ohtani Y, et al. c-Myc is an important direct target of Notch1 in T-cell acute lymphoblastic leukemia/lymphoma. Genes Dev. 2006; 20(15):2096-2109. [PubMed: 16847353]

34. Swiatek PJ, Lindsell CE, del Amo FF, Weinmaster G, Gridley T. Notch1 is essential for postimplantation development in mice. Genes Dev. 1994; 8(6):707-719. [PubMed: 7926761] . * Description of Notch-1 knockout mice phenotype.

35. Hamada Y, Kadokawa Y, Okabe M, Ikawa M, Coleman JR, Tsujimoto Y. Mutation in ankyrin repeats of the mouse Notch2 gene induces early embryonic lethality. Development. 1999; 126(15): 3415-3424. [PubMed: 10393120]

36. Fan X, Mikolaenko I, Elhassan I, et al. Notch1 and notch2 have opposite effects on embryonal brain tumor growth. Cancer Res. 2004; 64(21):7787-7793. [PubMed: 15520184]

37. Ito T, Udaka N, Yazawa T, et al. Basic helix-loop-helix transcription factors regulate the neuroendocrine differentiation of fetal mouse pulmonary epithelium. Development. 2000; 127(18): 3913-3921. [PubMed: 10952889] 
38. Sriuranpong V, Borges MW, Ravi RK, et al. Notch signaling induces cell cycle arrest in small cell lung cancer cells. Cancer Res. 2001; 61(7):3200-3205. [PubMed: 11306509]

39. Nakakura EK, Sriuranpong VR, Kunnimalaiyaan M, et al. Regulation of neuroendocrine differentiation in gastrointestinal carcinoid tumor cells by notch signaling. J. Clin. Endocrinol. Metab. 2005; 90(7):4350-4356. [PubMed: 15870121]

40. Kunnimalaiyaan M, Yan S, Wong F, Zhang YW, Chen H. Hairy Enhancer of Split-1 (HES-1), a Notch1 effector, inhibits the growth of carcinoid tumor cells. Surgery. 2005; 138(6):1137-1142. [PubMed: 16360401]

41. Zheng Q, Qin H, Zhang H, et al. Notch signaling inhibits growth of the human lung adenocarcinoma cell line A549. Oncol. Rep. 2007; 17(4):847-852. [PubMed: 17342326]

42. Jiang X, Zhou JH, Deng ZH, Qu XH, Jiang HY, Liu Y. Expression and significance of Notch1, Jagged 1 and VEGF in human non-small cell lung cancer. Zhong Nan Da Xue Xue Bao Yi Xue Ban. 2007; 32(6):1031-1036. [PubMed: 18182722]

43. Westhoff B, Colaluca IN, D'Ario G, et al. Alterations of the Notch pathway in lung cancer. Proc. Natl. Acad. Sci. U.S. A. 2009; 106(52):22293-22298. [PubMed: 20007775] . * First identification of Notch-1 activating mutations in NSCLC.

44. Baumgart A, Seidl S, Vlachou P, et al. ADAM17 regulates epidermal growth factor receptor expression through the activation of Notch1 in non-small cell lung cancer. Cancer Res. 70(13): 5368-5378. [PubMed: 20551051]

45. Dang TP, Gazdar AF, Virmani AK, et al. Chromosome 19 translocation, overexpression of Notch3, and human lung cancer. J. Natl. Cancer Inst. 2000; 92(16):1355-1357. [PubMed: 10944559]

46. Kees UR, Mulcahy MT, Willoughby ML. Intrathoracic carcinoma in an 11-year-old girl showing a translocation t(15;19). Am. J. Pediatr. Hematol. Oncol. 1991; 13(4):459-464. [PubMed: 1785673]

47. Lee AC, Kwong YI, Fu KH, Chan GC, Ma L, Lau YL. Disseminated mediastinal carcinoma with chromosomal translocation (15;19). A distinctive clinicopathologic syndrome. Cancer. 1993; 72(7):2273-2276. [PubMed: 8374886]

48. Kubonishi I, Takehara N, Iwata J, et al. Novel t(15;19)(q15;p13) chromosome abnormality in a thymic carcinoma. Cancer Res. 1991; 51(12):3327-3328. [PubMed: 2040007]

49. Haruki N, Kawaguchi KS, Eichenberger S, et al. Dominant-negative Notch3 receptor inhibits mitogen-activated protein kinase pathway and the growth of human lung cancers. Cancer Res. 2005; 65(9):3555-3561. [PubMed: 15867348]

50. Konishi J, Yi F, Chen X, Vo H, Carbone DP, Dang TP. Notch3 cooperates with the EGFR pathway to modulate apoptosis through the induction of bim. Oncogene. 2010; 29(4):589-596. [PubMed: 19881544]

51. Costa DB, Halmos B, Kumar A, et al. BIM mediates EGFR tyrosine kinase inhibitor-induced apoptosis in lung cancers with oncogenic EGFR mutations. PLoS Med. 2007; 4(10):1669-1679. [PubMed: 17973572]

52. Konishi J, Kawaguchi KS, Vo H, et al. Gamma-secretase inhibitor prevents Notch3 activation and reduces proliferation in human lung cancers. Cancer Res. 2007; 67(17):8051-8057. [PubMed: 17804716]

53. Lin L, Mernaugh R, Yi F, Blum D, Carbone DP, Dang TP. Targeting specific regions of the Notch3 ligand-binding domain induces apoptosis and inhibits tumor growth in lung cancer. Cancer Res. 2010; 70(2):632-638. [PubMed: 20068176]

54. Dang TP, Eichenberger S, Gonzalez A, Olson S, Carbone DP. Constitutive activation of Notch3 inhibits terminal epithelial differentiation in lungs of transgenic mice. Oncogene. 2003; 22(13): 1988-1997. [PubMed: 12673204]

55. Moellering RE, Cornejo M, Davis TN, et al. Direct inhibition of the NOTCH transcription factor complex. Nature. 2009; 462(7270):182-188. [PubMed: 19907488] . * Novel approach to Notch inhibition.

56. Wu Y, Cain-Hom C, Choy L, et al. Therapeutic antibody targeting of individual Notch receptors. Nature. 2010; 464(7291):1052-1057. [PubMed: 20393564] 
57. Riccio O, van Gijn ME, Bezdek AC, et al. Loss of intestinal crypt progenitor cells owing to inactivation of both Notch1 and Notch2 is accompanied by derepression of CDK inhibitors p27Kip1 and p57Kip2. EMBO Rep. 2008; 9(4):377-383. [PubMed: 18274550] 


\section{Key issues}

- The current standard of care to treat advanced NSCLC remains doublets including cytotoxic compounds, taxols, nucleoside analogs, antimetabolites and other classes of pharmaceuticals. Almost invariably, the tumor will recur and become therapy insensitive.

- Hypoxic tumor microenvironment appears to be a major culprit in chemotherapy resistance because of poor efficacy of current drugs in such environment, and because hypoxic tumor areas provide a niche to CSC.

- Notch signaling (especially Notch-1 signaling) plays a major role in determining NSCLC cell survival in hypoxia, and is implicated in CSC renewal.

- Targeting Notch signaling in NSCLC appears a particularly attractive therapeutic strategy because of the above-mentioned reasons.

- Gamma-secretase inhibitors have the potential to be tested in the clinic in the very near future despite their toxicity. Other avenues to tackle Notch signaling in cancer are in continuous development. 

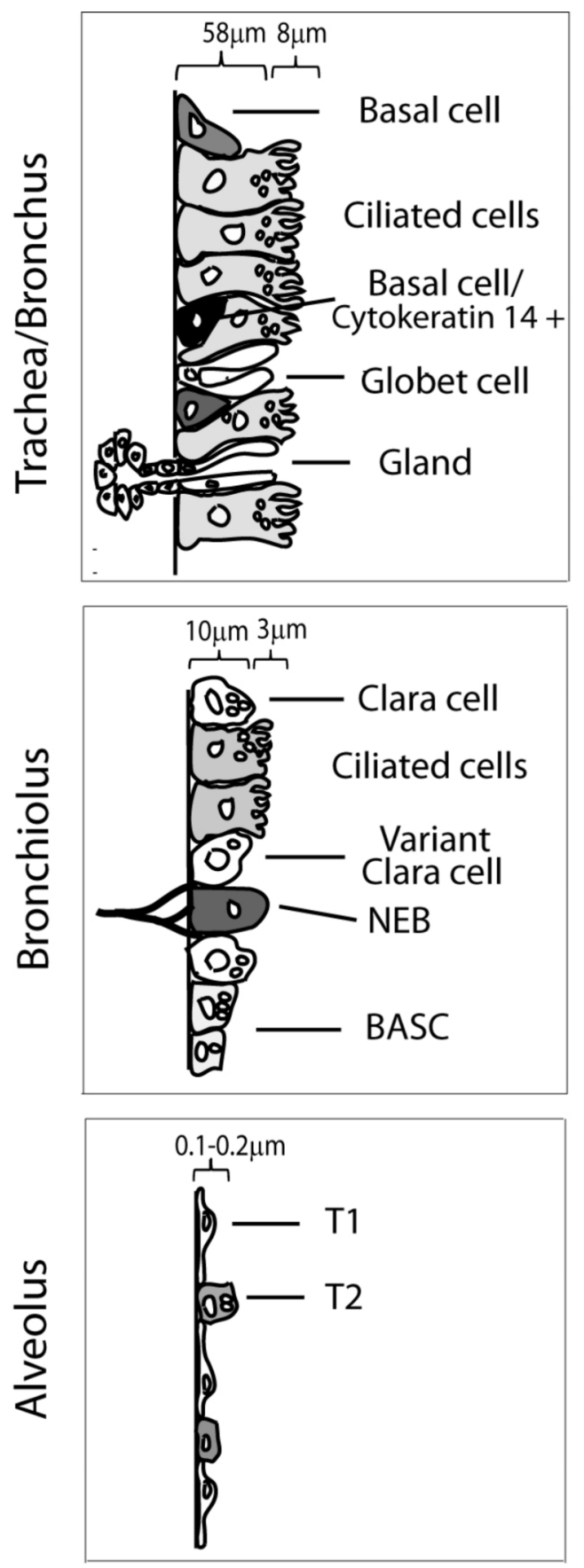

Figure 1.

Architecture of the airways epithelium. Abbreviations: NEB, neuroepithelial body; BASC, bronchioalveolar stem cell; T1, pneumocyte of type 1; T2, pneumocyte of type 2 . 


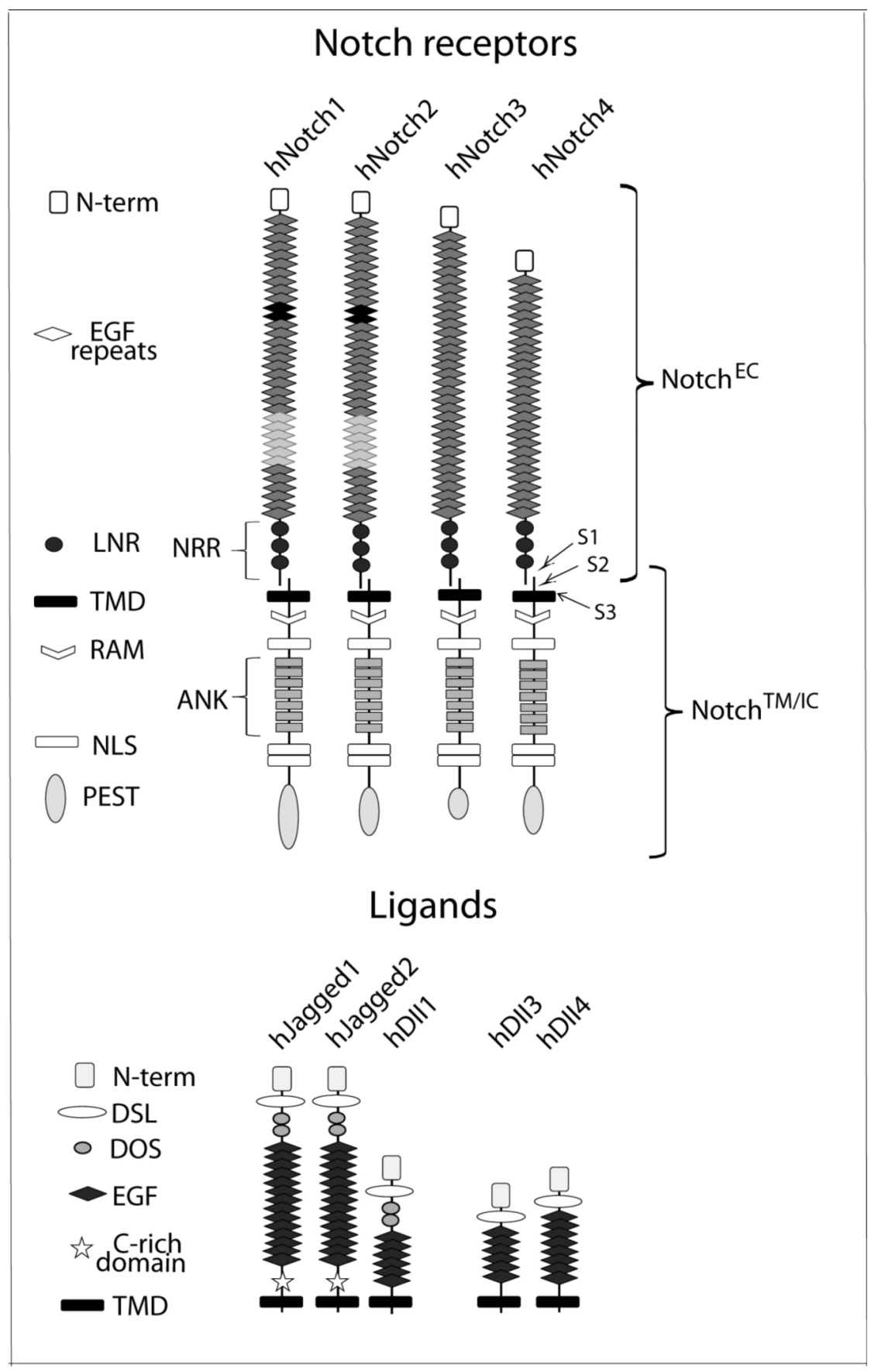

Figure 2.

Structure of human (h) Notch receptors and ligands.

Notch receptors are heterodimeric, single pass transmembrane proteins. The extracellular portion of Notch (Notch ${ }^{\mathrm{EC}}$ ) is non-covalently bound to the C-terminal, transmembrane portion of Notch (Notch ${ }^{\mathrm{TM}}$ ). Notch ${ }^{\mathrm{EC}}$ is a modular polypeptide containing a variable (among different receptors) number of epidermal growth factor (EGF)-like repeats. Repeats drawn in black in Notch-1 and -2 are those responsible for receptor-ligand interaction. The ligand interaction region of Notch-3 and -4 has not been determined yet. EGF repeats are targeted for glycosylation and fucosylation during protein maturation. Downstream from the EGF repeats are three evolutionarily conserved domains called Lin12/Notch repeats (LNR). 
Alongside the region immediately following the LNR, the heterodimerization domain, this portion of Notch ${ }^{\mathrm{EC}}$ hides the N-terminal region of Notch ${ }^{\mathrm{TM}}$ from ADAM cleavage, and is therefore referred as negative regulatory region (NRR). Upon ligand binding, the NRR is pulled away from Notch ${ }^{\mathrm{TM}}$ allowing ADAM cleavage at site 2 (S2), an event followed by $\gamma$-secretase cleavage at site 3 (S3) within the transmembrane domain (TMD). S1 is the site of furin-like convertase constitutive cleavage during Notch maturation. After cut at S3, Notch $^{\text {IC }}$ is released. Notch ${ }^{\mathrm{IC}}$ comprises a RAM domain, responsible for Notch $^{\mathrm{IC}}$ association with CBF-1, which is followed by a nuclear localization sequence (NLS). Downstream are found seven ankyrin repeats (AR) that are thought to mediate interaction with Mastermindlike proteins. The C-terminal portion of Notch ${ }^{\mathrm{IC}}$ is believed to include the transcription activation domain, which also contains two additional NLS and a proline/glutamic acid/ serine/threonine rich motif (PEST) involved in the regulation of Notch degradation. Ligands are monomeric, single pass transmembrane proteins that share a conserved Delta/Serrate/ Lag-2 (DSL) domain followed by two Delta and OSM-11-like (DOS) domains (with the exception of Delta-3 and -4), a number of EGF repeats (which varies among different ligands), a cysteine-rich domain in Jagged-1 and -2, a TMD and a C-terminal portion. 